\title{
COVID-19 AND MUCORMYCOSIS (BLACK FUNGUS): AN EPIDEMIC WITHIN THE PANDEMIC
}

\author{
Ramandeep Singh Gambhir ${ }^{1}$, Amit Aggarwal ${ }^{2}$, Arvind Bhardwaj $^{3}$, Avineet Kaur $^{4}$, Ramandeep Kaur Sohi $^{5}$, Sonia Mehta ${ }^{6}$
}

${ }^{1}$ Department of Public Health Dentistry, Rayat and Bahra Dental College and Hospital, Mohali, India

${ }^{2}$ Department of Oral Medicine and Radiology, MM College of Dental Sciences and Research, MM

(Deemed to be University), Mullana, India

${ }^{3}$ Department of Periodontology and Oral Implantology, MM College of Dental Sciences and Research, MM (Deemed to be University), Mullana, India

${ }^{4}$ Department of Periodontology and Oral Implantology, Swami Devi Dyal Dental College and Hospital, Haryana, India

${ }^{5}$ Department of Public Health Dentistry, Sri Sukhmani Dental College and Hospital, Derabassi, Punjab, India

${ }^{6}$ Department of Microbiology, Dr BR Ambedkar State Institute of Medical Sciences (AIMS), Mohali, India

\begin{abstract}
The second wave of the COVID-19 pandemic has affected India significantly with country reporting more than 400,000 cases in the month of May 2021 and health system almost collapsing. This was attributed to the new mutant strain also called as the 'Delta Strain' which led to high surge of cases across the country. As the country was stabilising over this situation, another imminent threat in the form of Covid Associated Mucormycosis (CAM) challenged the already burdened health system of India. Also called as 'Black Fungus,' cases of CAM began to rise rapidly in the last week of May 2021 with multiple states reporting steady rise in the number of cases. Based on the published literature, India contributed to approximately 71\% of global cases of CAM from December 2019 to start of April 2021, with majority of the cases occurring during the second wave. The present paper focuses on the epidemic of CAM during the second wave in India highlighting the causes, symptoms and various treatment modalities that have been adopted to cure the disease. Also, spotlight has also been thrown on some other nations where cases of CAM have begun to emerge. Some key recommendations are also mentioned which can prove vital towards disease prevention.
\end{abstract}

Key words: COVID-19, SARS-CoV-2 pandemic, mucormycosis, India, diabetes, Black fungus

\section{INTRODUCTION}

The Novel Coronavirus disease (COVID-19, SARS-CoV-2), the deadliest pandemic of the millennium has brought the health system of the world down on its knees with millions of people falling prey to this deadly disease. Be a developing country or a developed country, countries all over the world were forced to impose a lock down as a measure of social distancing to curtail the spread of infection [10]. Wearing a face mask has become a new normal to prevent harmful aerosols from entering our respiratory system and causing the deadly infection [5]. According to the latest statistics from the World Health Organization (WHO), globally there are 176,303,596 confirmed COVID-19 cases and 3,820,026 deaths [19]. Currently, renowned pharmaceutical companies have developed vaccines (using different technologies) to protect people from this deadly disease and the process of vaccination is being carried out on priority basis by each and every nation [3]. However, still there is a large gap between the production of vaccines and population to be vaccinated. Hardly the world had emerged from the first wave of COVID-19, many countries including India experienced the second wave of the pandemic in March 2021, which was more deadlier than the first with social distancing measures failing to reduce rapidly increasing case numbers [1]. This was attributed because of the emerging triple mutant strains of the virus with high surge of cases on daily basis crippling the health system of the country [17]. According to WHO, only B.1.617.2, one of the three strains of the B.1.617, first detected in India, is a Variant of Concern (VOC) with greater

Corresponding author: Ramandeep Singh Gambhir, Department of Public Health Dentistry, Rayat and Bahra Dental College and Hospital, Mohali, Punjab, India PIN-140103, Tel.+91-99156-46007, e-mail: raman2g@yahoo.com

(C) Copyright by the National Institute of Public Health NIH - National Research Institute 
public health risks currently associated with this strain and it has already been reported in 85 countries globally and continues to be reported in new countries across all WHO regions. This is now labelled as the 'Delta Variant' [19]. This has made the virus more transmissible and immune to vaccines or makes it cause more severe disease. As per latest reports, a new variant called as 'Delta Plus Variant' has been reported in some states in India. This variant, in addition to its increased transmissibility, has got stronger binding to receptors of lung cells and potential reduction in monoclonal antibody response [20]. Moreover, the country witnessed a heavy demand of medical oxygen and post COVID cardiac complications during the second wave owing to the increasing number of cases because of the new variant.

To make the situation worse, India witnessed another epidemic, that of mucormycosis (Black fungus) amidst the COVID-19 pandemic during the second wave in May 2021. More than 31,000 cases have already been reported so far across the country and more than 2100 people have died as a result of mucormycosis [8]. Majority of cases have been reported from the state of Maharashtra followed by Rajasthan, Gujarat, Madhya Pradesh, Haryana, Delhi and Punjab. Cases of mucormycosis have more than doubled in India in late 2020 compared to the corresponding months of 2019 establishing a direct correlation between COVID-19 and lethal black fungus viral infection now ravaging in almost each and every state of the country [4].

\section{CAUSE OF MUCORMYCOSIS (BLACK FUNGUS)}

According to the present data, Rhizopus arrhizus is the predominating agent causing Covid-associated Mucormycosis (CAM) in India [8]. It can be described as "black fungus," because it makes infected tissues turn black. Most mucormycosis infections are lifethreatening, and various risk factors, such as diabetic ketoacidosis and neutropenia are present in most cases which can lead to further complications [15]. A majority of patients of CAM have uncontrolled diabetes. Apart from the fact that blood sugar levels are elevated with steroids, COVID-19 itself can induce damage to pancreatic islet cells. Blood sugar levels may also get elevated because of increased resistance to insulin due to the inflammatory reaction. This suggests that indiscriminate and prolonged use of steroids by doctors treating mild COVID-19 patients is the key factor in the development of mucormycosis. According to the guidelines, steroids are recommended only in moderate to severe cases of COVID-19 when oxygen levels drop [8]. Rhino-orbital-cerebral mucormycosis (ROCM) is the most common form of mucormycosis in patients suffering from diabetes mellitus. The infection develops after inhalation of fungal sporangia spores into the paranasal sinuses [4].

According to an advisory issued by the Indian Council of Medical Research, the following conditions in COVID-19 patients increase the risk of mucormycosis infection [14]:

- People with uncontrolled diabetes

- Weakening of immune system due to excessive use of steroids

- Prolonged Intensive Care Unit (ICU) stay / hospital stay

- Co-morbidities / post organ transplant / cancer

- Patients on Voriconazole therapy (used to treat serious fungal infections)

\section{TWO-FOLD RISE IN MUCORMYCOSIS CASES IN A YEAR}

According to reports of a multi-centre study conducted in 16 Indian hospitals involving both CAM and non-Covid mucormycosis (non-CAM), it was found that overall mucormycosis case fatality rate was 45.7 percent at 12 weeks [12]. It was also found that newly detected diabetes mellitus was more frequent during the evaluation of mucormycosis among CAM patients that non-CAM patients suggesting that many COVID infected patients may not have been aware of their diabetic condition when diagnosed with black fungus. India is called as diabetes capital of the world; the indiscriminate and non-judicious use of steroids in patients suffering from diabetes potentially led to the surge of mucormycosis in the country. It was reported that there was 2.1-fold rise in mucormycosis cases during the study period compared with 2019 [12].

\section{CLINICAL FORMS AND SYMPTOMS OF MUCORMYCOSIS}

On the basis of the anatomical site of involvement, there are various clinical forms of mucormycosis reported in various case series from India. ROCM is the commonest form (45-74\%), followed by cutaneous (10-31\%), pulmonary (3-22\%), renal $(0.5-9 \%)$, gastrointestinal $(2-8 \%)$, and disseminated infections $(0.5-9 \%)$. Breast, ear, spine, heart and bone infections are other unusual sites of infection reported in the literature from India [13].

Mucormycosis begins to manifest as skin infection in the air pockets located behind our forehead, nose, cheekbones, and in between the eyes and teeth [13]. Then it involves the eyes and lungs can even spread to the brain. It leads to blackening or discoloration over the nose, blurred or double vision, chest pain, breathing difficulties and coughing of blood particularly in case of pulmonary mucormycosis (Figure 1). Patients with disseminated infection in the brain can develop mental 


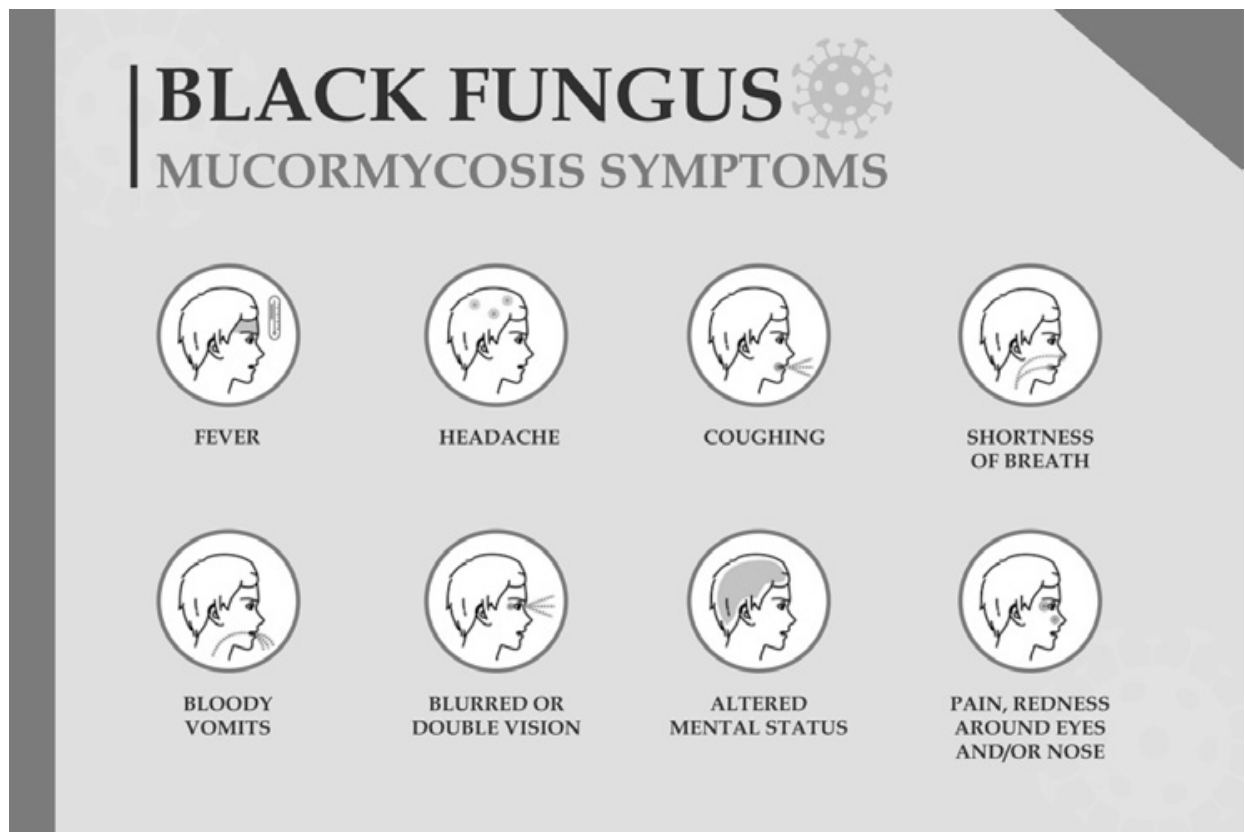

Figure 1. Symptoms of Mucormycosis (Black Fungus)

status changes or coma. According to the advisory issued by the Indian Council of Medical Research, not all cases of blocked nose should be considered as cases of bacterial sinusitis, particularly during/ after the treatment of Covid-19 patients. According to medical specialists, patients report to the hospital late considering these symptoms as normal which leads to further complications. The mean duration between diagnosis of COVID-19 and onset of mucormycosis symptoms is approximately $15.6 \pm 9.6$ days. A delay of even 6 days in seeking treatment doubles the 30 day mortality from $35 \%$ to $66 \%$ [16].

\section{MANAGEMENT OF MUCORMYCOSIS}

The treatment of mucormycosis starts with the early diagnosis and initiation of therapy along with the surgical debridement of the infected tissue, antifungal therapy, and managing the underlying disease. The first-line drug of choice is Amphotericin B (AmB) and later on posaconazole and isavuconazole are prescribed depending on the condition of the patient [6]. Gap in treatment protocol and the financial constraints of patients to afford liposomal AmB are the major drawbacks in managing patients suffering from mucormycosis in India. Moreover, due to the rapid surge of mucormycosis cases in India, there is shortage of AmB.

Different modes of therapy and mortality rates in Indian population are depicted in Figure 2 (it should be duly noted that data shown is based on different studies shown in the main article) [13]. According to existing data, mortality rate was low in patients treated with a combination of $\mathrm{AmB}$ and surgical

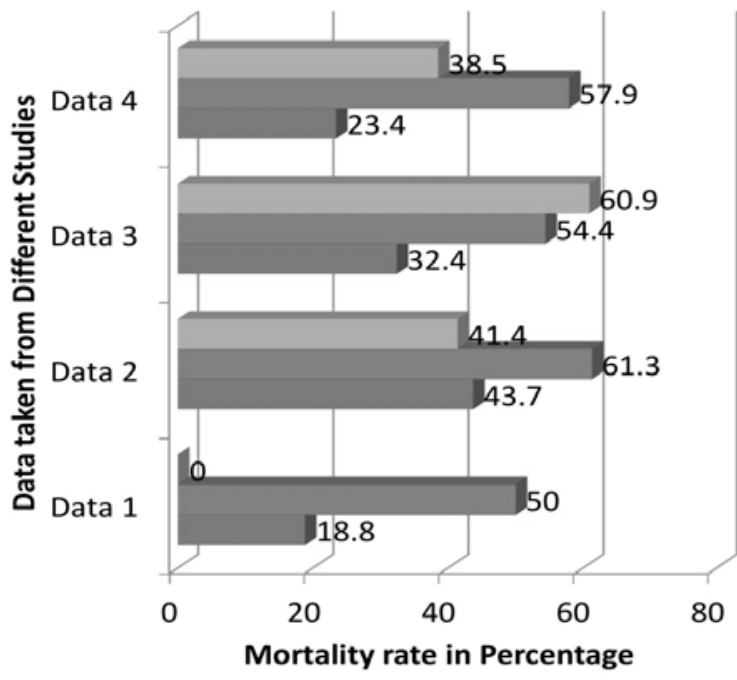

Eurgery alone

amphotericin B alone

Amphotericin B + Surgery

Figure 2. Different modes of therapy and mortality rates in Indian population 
debridement of the infected tissue (19-44\%) compared with AmB monotherapy (50-61\%). These findings are in congruence with the global data [7]. Posaconazole and isavuconazole were used as salvage therapy in the treatment of mucormycosis. A study assessing the safety and efficacy of posaconazole in ROCM patients was conducted in South India. The study findings showed no mortality; there was complete resolution of the disease in $66.6 \%$ of the patients and remaining patients reported significant reduction of the disease [9]. The new anti-Mucorales drug, isavuconazole proved to be equally effective as $\mathrm{AmB}$, however, it has been recently introduced in Indian market and its efficacy is still to be assessed in Indian population.

Surgical removal of the infected (necrotic) tissue, debridement, and enucleation of eye (if involved), also proves to be lifesaving. Other clinical interventions like proper cleaning and sterilization of humidifiers and ventilators, utilization of disposable or disinfected personal protective equipment, and proper hygiene maintenance are also equally important in disease prevention [4]. It is also recommended that everyone should follow pristine oral hygiene measures like regular tooth brushing, not using a common holder and disinfecting the toothbrush each time after use by dipping it in some antiseptic mouthwash. One should also dispose the older toothbrush post recovery from COVID-19.

\section{CASES OF MUCORMYCOSIS REPORTED IN THE AMERICAS AND EUROPE}

Seven countries in the Region of the Americas have reported to $\mathrm{PAHO} / \mathrm{WHO}$ or published the detection of 16 CAM cases till $9^{\text {th }}$ June 2021 [11]. These include Brazil, Chile, Honduras, Mexico, Paraguay, Uruguay and the United States of America. Majority of the cases have clinical presentation in the form of ROCM. Diabetes mellitus has been found to be the main underlying disease or co-morbidity in more than $95 \%$ of cases apart from other diseases like atypical pneumonia, arterial hypertension etc. Cases have also been reported from other regions like United Kingdom and Italy. Four cases of CAM have also been reported from Netherlands between December 2020 and May 2021 of which three developed the infection during intensive care unit. admission and three lost their lives [2]. The Pan American Health Organization / World Health Organization (PAHO/WHO) has urged Member States to prepare their respective health services to increase clinical suspicion of mucormycosis in patients with COVID-19, especially those with comorbidities and treatment with corticosteroids and other immunosuppresants.

\section{CASES OF MUCORMYCOSIS IN NEPAL (INDIA'S NEIGHBOUR)}

This Himalayan nation and India's immediate neighbour has reported 11 cases of CAM and two people have lost their lives due to this infection [18]. It has been reported that there were only a limited number of mucormycosis cases previously but cases grew rapidly among COVID-19 patients during the second wave of the pandemic which began in early April 2021. There is also shortage of drugs likes AmB and Deoxycholate (used to treat black fungus infection) in the Nepali market and now the Government of Nepal is procuring these drugs from international market and WHO.

\section{CONCLUSION AND KEY RECOMMENDATIONS}

Following the surge of COVID-19 associated mucormycosis and the Government of India directive, mucormycosis was put under the category of 'notifiable disease' by several states in May 2021. This will provide better insights into the disease burden, population characteristic, risk factors, clinical spectrum and outcomes of these patients. This was also important to check the shortage and black marketing of the drug Amphotericine-B as price of the drug rose exponentially due to the rapid demand. Our findings emphasize the need to be aware of invasive mucormycosis developing in COVID-19 patients, especially while receiving corticosteroids, including patients without (poorly controlled) diabetes mellitus and outside the ICU. An aggressive approach should be employed when invasive mucormycosis is diagnosed, including early surgery and targeted antifungal treatment. Patients receiving oxygen therapy should ensure that the water in the humidifier is clean and is refilled regularly. As fungus can breed on wet surfaces, it should be ensured that there is no leakage of water. Even after recovering from COVID-19, one should not miss any warning signs and symptoms described in the text, as the fungal infection can emerge even weeks or months after recovery. Steroids should be used wisely according to the doctor's advice.

Mass vaccination of the population, sharing health information, building community trust etc. are vital primary health care measures which can protect everyone during COVID-19 (World Health Organization) (Figure 3) [19]. 


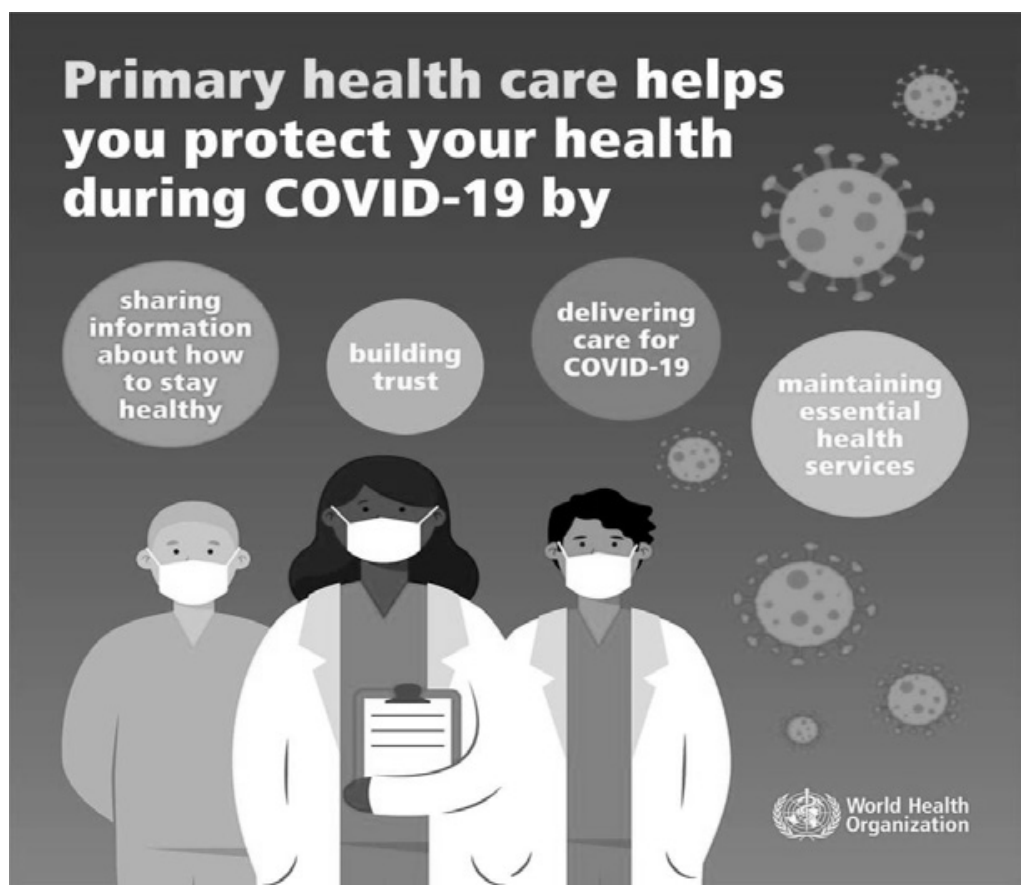

Figure 3. Primary health care measures to protect from COVID-19 according to WHO [19]

\section{REFERENCES}

1. Atreya A.: Death and dignity in India and Nepal during the second wave of Coronavirus disease 2019. Med Sci Law. 2021:258024211021384. doi: 10.1177/00258024211021384.

2. Buil J.B., van Zanten A.R.H., Bentvelsen R.G., Rijpstra T.A., Goorhuis B., van der Voort S., Wammes L.J., Janson J.A., Melchers M., Heusinkveld M., Melchers W.J.G., Kuijper E.J., Verweij PE.: Case series of four secondary mucormycosis infections in COVID-19 patients, the Netherlands, December 2020 to May 2021. Euro Surveill. 2021;26. doi: 10.2807/1560-7917. ES.2021.26.23.2100510.

3. Carneiro D.C., Sousa J.D., Monteiro-Cunha J.P.: The COVID-19 vaccine development: A pandemic paradigm. Virus Res. 2021;301:198454. doi: 10.1016/j. virusres.

4. Christopher N Rocha I., Mehedi Hasan M., Goyal S., Patel T., Jain S., Ghosh A., Denise D Cedeño T.: COVID-19 and Mucormycosis Syndemic: Double Health Threat to a Collapsing Healthcare System in India. Trop Med Int Health. 2021. doi: 10.1111/tmi.13641.

5. Forouzandeh P., O'Dowd K., Pillai S.C.: Face masks and respirators in the fight against the COVID-19 pandemic: An overview of the standards and testing methods. Saf Sci. 2021;133:104995. doi: 10.1016/j.ssci.2020.104995.

6. Imran M., A S A., Tauseef M., Khan S.A., Hudu S.A., Abida.: Mucormycosis medications: a patent review. Expert Opin Ther Pat. 2021. doi: 10.1080/13543776.2021.1939308.

7. Jeong W., Keighley C., Wolfe R., Lee W.L., Slavin M.A., Chen S.C., Kong D.C.M.: Contemporary management and clinical outcomes of mucormycosis: A systematic review and meta-analysis of case reports. Int $\mathrm{J}$ Antimicrob Agents. 2019;53:589-97.
8. Looking beyond the second wave of Covid. Available from: www.epapertribuneindia.com. Accessed on: $12^{\text {th }}$ June 2021.

9. Manesh A., John A.O., Mathew B., Varghese L., Rupa V., Zachariah A., Varghese G.M.: Posaconazole: an emerging therapeutic option for invasive rhino-orbitocerebral mucormycosis. Mycoses. 2016;59:765-772.

10. Onyeaka H., Anumudu C.K., Al-Sharify Z.T., EgeleGodswill E., Mbaegbu P.: COVID-19 pandemic: A review of the global lockdown and its far-reaching effects. Sci Prog. 2021;104:368504211019854. doi: $10.1177 / 00368504211019854$.

11. Pan American Health Organization / World Health Organization. Epidemiological Alert: COVID-19 associated Mucormycosis. 11 June 2021, Washington, D.C.: PAHO/WHO; 2021. Available from: www.paho. org. Accessed on: $17^{\text {th }}$ June 2021.

12. Patel A., Agarwal R., Rudramurthy S.M., Shevkani M., Xess I., Sharma R., Savio J., Sethuraman N., Madan S., Shastri P., Thangaraju D., Marak R., Tadepalli K., Savaj P., Sunavala A., Gupta N., Singhal T., Muthu V., Chakrabarti A., MucoCovi Network3.: Multicenter Epidemiologic Study of Coronavirus DiseaseAssociated Mucormycosis, India. Emerg Infect Dis. 2021;27. doi: 10.3201/eid2709.210934.

13. Prakash H., Chakrabarti A.: Epidemiology of Mucormycosis in India. Microorganisms. 2021;9:523. doi: 10.3390/microorganisms9030523.

14. Press Information Bureau, Government of India. Available from: https://pib.gov.in/PressReleseDetailm. aspx?PRID=1718501. Accessed on: $15^{\text {th }}$ June 2021 .

15. Sahota R., Gambhir R., Anand S., Dixit A.: Rhinocerebral Mucormycosis: Report of a Rare Case. Ethiop J Health Sci. 2017;27:85-90. 
16. Sen M., Lahane S., Lahane T.P., Parekh R., Honavar S.G.: Mucor in a Viral Land: A Tale of Two Pathogens. Indian J Ophthalmol. 2021;69:244-52.

17. Triple-mutant 'Bengal strain' a new worry in second wave. Times of India. Available from: www. timesofindia.com. Accessed on: $14^{\text {th }}$ June 2021.

18. Two dead and 11 suffering from black fungus in Nepal. Available from: https://imesofindia.indiatimes.com/ world/south-asia. Accessed on: 18th June 2021.
19. WHO. Coronavirus (COVID-19) Dashboard. Available from: covid19.who.int. Accessed on: $28^{\text {th }}$ June, 2021.

20. 51 Delta plus cases across 12 states, 2 in Punjab, 1 in Haryana. Available from: www.epapertribuneindia. com. Accessed on: $24^{\text {th }}$ June 2021.

Received: 18.06.2021

Accepted: 29.06.2021

Published online first: 07.07.2021 